\title{
La calidad periodística en España según la percepción de los periodistas
}

\author{
Josep Lluís GÓMEZ MOMPART \\ Universitat de València \\ josep.11.gomez@uv.es \\ Juan Francisco GuTiÉRREZ LozANO \\ Universidad de Málaga \\ jfg@uma.es \\ Dolors Palau SAmPio \\ Universitat de València \\ dolors.palau@uv.es
}

Recibido: 5 de diciembre de 2014

Aceptado: 8 de mayo de 2015

\section{Resumen}

Este artículo expone los resultados de una investigación sobre la percepción que tienen los periodistas españoles de la calidad periodística, teniendo en cuenta el contexto económico, tecnológico, empresarial, laboral y profesional. Los resultados de una encuesta, en la que participan 363 periodistas españoles con al menos tres años de experiencia en diferentes medios, ponen de manifiesto carencias relativas tanto a la relevancia y diversidad de los temas abordados como al acceso a las fuentes o el tratamiento y verificación de los datos, así como a las condiciones laborales o a los retos derivados de la adaptación a las nuevas tecnologías y a las imposiciones de la acuciante inmediatez. Junto a este retrato general, también se observan visiones particulares en función del medio, los años de experiencia laboral o el cargo ocupado.

Palabras clave: calidad, percepción, valores profesionales, periodistas.

\section{Journalistic quality in Spain according to the perception of the journalists}

\begin{abstract}
This article discusses the results of a research on the perception of the journalistic quality by Spanish journalists, taking into account the economical and technological context. The survey, which involved 363 Spanish journalists with at least three years of experience in different media, reveals gaps relating not only to the relevance and the diversity of the topics covered, but also relating to the access to the sources or the treatment and verification of the data, as well as the working conditions or to the challenges arising from the adaptation to new technologies and to the impositions of the immediacy. Together with this portrait general, there are also particular viewpoints depending on the media, the work experience or the post occupied.
\end{abstract}

Keywords: quality, perception, professional values, journalists.

\section{Referencia normalizada}

GÓMEZ MOMPART, Josep Lluís; GUTIÉRREZ LOZANO, Juan Francisco; y PALAU SAMPIO, Dolors (2015): "La calidad periodística en España según la percepción de los periodistas". Estudios sobre el Mensaje Periodístico. Especial noviembre "Periodismo e información de calidad", págs.: 13-30. Madrid, Servicio de Publicaciones de la Universidad Complutense.

Sumario: 1. Introducción. 2. Metodologías para el estudio de la calidad periodística; 2.1. Sobre la empresa y el producto; 2.2. Sobre el producto y el periodista; 2.3. Sobre el periodista y el entorno socioprofesional; 2.4. Sobre la pérdida de calidad. 3. Análisis de los resultados; 3.1. Relevancia de las informaciones y producción propia; 3.2. Investigación, proximidad y fuentes documentales 3.3. Facticidad y equidad; 3.4. Un contexto laboral poco propicio; 3.5. Adaptación a las nuevas tecnologías e inmediatez. 4. Conclusiones. 5. Referencias bibliográficas. 


\section{Introducción}

El número y el tono de las críticas hacia la profesión periodística ha arreciado en los últimos años, hasta el punto de situarla como una de las menos valoradas, como han puesto de manifiesto encuestas del Centro de Investigaciones Sociológicas (2013) ${ }^{1}$. Este debate, que coincide con una crisis económica y de modelo de negocio de proporciones insólitas, convierte en inevitable preguntar a los profesionales qué percepción tienen acerca del trabajo que realizan, con el objetivo de poder indagar mejor en las causas que motivan la nefasta valoración ciudadana. Esta cuestión se antoja clave desde el punto de vista de la responsabilidad social que entraña el ejercicio de la profesión periodística, cuya buena praxis influye en la salud democrática.

Este artículo recoge los resultados de una encuesta realizada a periodistas que cuentan con más de tres años de experiencia profesional, con distintas responsabilidades y vinculados tanto a medios de comunicación convencionales como digitales. El análisis recoge los datos extraídos de una encuesta de 45 preguntas (38 cerradas y 7 abiertas), realizada con la colaboración de las asociaciones y colegios profesionales. Se llevó a cabo durante los meses de junio y julio de 2013 y fue respondida anónimamente por 363 periodistas ( $45 \%$ mujeres y $55 \%$ hombres). El objetivo era conocer cómo los protagonistas de la información evalúan su propio trabajo y en base a qué criterios valoran su calidad, mediante una metodología de trabajo que combina un acercamiento cuantitativo y cualitativo al objeto de estudio.

La encuesta se organizó en base a cuatro ejes fundamentales: la estructura y el comportamiento del medio (17 preguntas), el tratamiento de la información (10 preguntas), el cuidado y esmero productivos (11 preguntas), y las causas y razones de la pérdida de calidad ( 7 cuestiones generales). En este último apartado se combinaron las respuestas cerradas y abiertas, con el objetivo de permitir a los encuestados añadir nuevos elementos a tener en cuenta a la hora de perfilar cómo se percibe la calidad periodística e incluir apreciaciones que hubieran podido quedar fuera del cuestionario.

La investigación arroja luz sobre cuáles son los criterios que los periodistas españoles asocian a un trabajo de calidad y nos permite conocer sus reflexiones acerca de la distancia existente entre la realidad a la que se enfrentan en su día a día y el horizonte de excelencia que barajan como ideal.

\section{Metodologías para el estudio de la calidad periodística}

La preocupación por la calidad periodística arranca en el pasado siglo, vinculada a diferentes tradiciones metodológicas y objetivos de diversa índole. Si la investigación en el ámbito norteamericano ha tenido una orientación comercial, enmarcada en la búsqueda de beneficios económicos, la realizada en Alemania se ha desarrollado en

1 En su informe de marzo de 2013, señala que un 27,6\% de los ciudadanos muestra una confianza negativa (menos de 5 puntos de una escala de 10) respecto a los medios, mientras que casi la mitad (46,4\%) les concede una confianza mínima y un $21,7 \%$ declara un nivel de confianza notable ( 7 y 8). Esta desafección se traslada también a la valoración de la profesión, la más baja (un 6,4 sobre 10) -junto a la de juez-de una escala de diez propuestas, y en especial, a la hora de recomendarla a hijos o amigos, algo que solo osaría hacer el 1,7\%, apenas por delante de la de albañil y dos décimas por debajo de la de fontanero. 
torno al profesionalismo, aunque los países escandinavos fueron los pioneros en Europa, a partir de la noción de informatividad, una idea ligada a la cantidad de información y el espacio dedicado por un medio a un conjunto de hechos. La tercera línea, desarrollada en algunos países latinoamericanos -sin olvidar ciertas investigaciones de España-, ha centrado su atención en la responsabilidad social y la calidad democrática, al concebir el periodismo como un bien público de cuyo buen y honesto funcionamiento depende el bienestar y el desarrollo democrático.

Las propuestas sugeridas en las últimas décadas para el estudio de la calidad, tanto en Europa y América, parten del periodismo entendido ya como una práctica social institucionalizada moderna, circunscrita a sistemas políticos democráticos. El papel relevante que el periodismo juega dentro del campo de la comunicación, y su relación con la esfera política y su repercusión sobre la opinión pública, desencadena la necesidad de conocer en qué medida y de qué manera la información pública cumple con los requisitos que se le presuponen válidos y necesarios en una sociedad avanzada. Ello ha llevado a investigadores y organismos que velan por la buena práctica periodística a establecer métodos cualitativos de análisis, aunque para algunos aspectos, también cuantitativos.

Las principales propuestas que se han planteado estudiar la calidad periodística -como se expone ampliamente en el volumen La calidad periodística (Gómez Mompart, Gutiérrez y Palau, 2013)- abarcan desde registros que presentan evidencias de aspectos positivos sobre qué es y cómo debe ser el periodismo en una sociedad democrática hasta las metodologías y técnicas que analizan cuál es el periodismo que se ejerce en relación con las empresas informativas, el producto periodístico a partir de la práctica profesional, institucionalizada y con unas pautas determinadas, y finalmente el entorno donde se desarrolla esta importante actividad social o la percepción por parte de la ciudadanía.

La encuesta que enmarca esta investigación tiene como punto de partida los métodos de registro, análisis y evaluación (Gómez Mompart y Palau, 2013), que se inspiran en las teorías del gatekeeping y el newsmaking. En ellas prima, por un lado, la idea del profesional del periodismo como garante, como guardián que debe velar por el buen periodismo, dado que recibir una información veraz es un derecho fundamental de los ciudadanos en las sociedades abiertas y esta responsabilidad delegada recae en el periodista y, por supuesto, también en los patronos de los medios informativos. Y, por otro, la tesis de que la producción informativa debe responder a unos criterios de noticiabilidad basados en unos valores razonables de interés público, cuya fiabilidad entre el hecho y su relato debe ajustarse a unos principios básicos de veracidad, comprobación, contraste y honestidad.

El desarrollo a lo largo del siglo pasado de diversas teorías que escrutaban la función y el sentido de la información, de cuanto compone y supone el denominado campo periodístico y el habitus (práctica) profesional (interiorizada) según Pierre Bourdieu, evidenciaron las dificultades de establecer métodos para mesurar la calidad periodística. No obstante, las teorías que desentrañan los intereses y la organización de las empresas de medios de información, muestran los inconvenientes de las rutinas productivas o indagan en la cultura profesional, revelan la hegemonía de los definidores primarios, evidencian la instrumentalización de los contenidos o inspeccionan 
la estructura comunicativa ofrecen interesantes pistas para anotar -y después estudiar- aspectos cruciales del quehacer periodístico, así como sugerir indicios del signo $-\mathrm{e}$, incluso, en algunos casos, el porcentaje- de las desviaciones o de la deformación del periodismo entendido éste como producto, servicio, comercio o bien público.

Este amplio abanico, tanto de teorías de la comunicación como de métodos y técnicas inspirados explícita o implícitamente en ellas, bebe de disciplinas diversas, que incluyen desde la sociología a la economía, pasando por la lingüística, el derecho o la ciencia política aplicadas a la comunicación. Respecto a su formulación analítica, las metodologías para analizar la calidad periodística se ocupan particularmente de: i. la empresa y el producto, ii. el producto y el periodista, y iii. el periodista y el entorno socioprofesional. Esta división es la que guía los diferentes apartados de la encuesta, con punto final en el que se incide en las causas de la pérdida de la calidad.

La voluntad de centrarse en la "calidad periodística" y no sólo en la "calidad informativa" responde al interés de poner el acento, además de en el producto periodístico, en todo el proceso informativo-comunicativo, desde periodistas y editores a administradores de la empresa periodística. De aquí que haya que referirse, por un lado, a las propuestas que han incidido en considerar el periodismo como servicio o bien de consumo. Y, por otro, a aquellas otras que quieren apuntar a ideales de la calidad periodística, en cuanto a independencia, diversidad y objetividad, los cuales se sustentan en los valores democráticos y en aquellas condiciones que garantizan el libre ejercicio del periodismo: los recursos, el orden político y legal y los estándares profesionales.

\subsection{Sobre la empresa y el producto}

El primer apartado de la encuesta (Estructura y comportamiento del medio informativo) contempla algunos de los indicadores propuestos por John Merrill y Ralph Lowenstein en Media, Messages and Men (1971) y Leo Bogart en Press and public (1989), contrastados con los indicadores de los tres conjuntos de índices (laboral, periodístico y empresarial) descritos por José Manuel de Pablos y Concha Mateos Martín (2004), juntamente con el tesaurus de ética periodística, elaborado por el amplio equipo de investigación dirigido por Salvador Alsius (Alsius y Salgado, 2010), sintetizado en cuatro aspectos: veracidad, justicia, libertad y responsabilidad.

Los indicadores planteados para el primer bloque de la encuesta hacen referencia a: independencia del medio; libertad de la redacción; diversidad de columnistas; papel del empresario; estatuto de redacción; nivel de formación del periodista; estabilidad en el puesto de trabajo; salario justo; jornadas laborales racionales; porcentaje de publicidad; cumplimiento de normas éticas; respeto a la cláusula de conciencia; grado de transparencia en el procesamiento de la información; temas que se eluden; número de agencias informativas; número de redactores en relación con el volumen de trabajo y número habitual de redactores en temas de relevancia informativa.

\subsection{Sobre el producto y el periodista}

En el segundo apartado de la encuesta (Tratamiento de la información) se han tomado en cuenta ciertos aspectos del patrón de medición denominado "Valor Agregado Pe- 
riodístico" (VAP), desarrollado por investigadores de la Pontificia Universidad Católica de Chile (VV.AA., 2001), pero especialmente la concreción hecha posteriormente por María Teresa Téramo (2006), que fija una decena de estándares de Calidad de la Información Periodística (confiabilidad, relevancia, interés, proporción, adecuación, transparencia, claridad, comprensibilidad, integración e incidencia), juntamente con algunos aspectos del conjunto de cinco indicadores probado para los informativos de televisión por Estrella Israel y Ricardo Pomares (2013: 156-157), a saber: indicadores relacionados con la temática (relevancia y jerarquización de la noticia); criterios de selección (los valores noticia); indicadores relacionados con el tratamiento y la presentación de las informaciones; indicadores relacionados con los personajes, las fuentes y la diversidad intercultural; $y$, finalmente, indicadores espacio-temporales y de localización de las noticias.

A partir de las reflexiones anteriores, para este segundo bloque, el cuestionario va referido a: uso de bases documentales primarias; porcentaje de informaciones relevantes; porcentaje de temas propios; reportajes de producción propia; noticias que abordan realidades próximas; acceso directo a los acontecimientos de los que se informa; tratamiento de los protagonistas; calidad fotoperiodística; infografías y/o gráficos de elaboración propia y periodismo de investigación.

\subsection{Sobre el periodista y el entorno socioprofesional}

Para el tercer apartado -y parcialmente del cuarto bloque- de la encuesta (Cuidado y esmero productivos), se han recuperado algunos índices del VAP, así como de otros indicadores propuestos por la Red de Periodismo de Calidad de México (VV.AA., 2006), que hacen referencia, por un lado, a los principios que dependen directamente del trabajo del periodista, de la formación, el compromiso y la ética, y, por otro, a los principios que dependen del entorno del profesional. También, a determinados aspectos desarrollados en la tesis doctoral de María González (2011: 258-345), quien combina un análisis de los aspectos formales, destinado a detectar posibles defectos y errores técnicos en la presentación y transmisión, y un análisis de los contenidos que centra su atención en tres aspectos: la diversidad, la independencia (entre otras cuestiones el grado de facticidad, distinguiendo entre hechos, afirmaciones y conjeturas) y el proceso de elaboración informativo.

Este apartado de nuestra encuesta plantea los siguientes indicadores: calidad de la escritura/narración; corrección lingüística; lenguaje apropiado; facticidad de las informaciones; diversidad de temas, protagonistas y ámbitos; diversidad intercultural; frecuencia de citas; pluralidad de fuentes; verificación de los datos ofrecidos; porcentaje de fuentes institucionales y/o corporativas; y enfoque predominante si es más 'emotivo'o 'informativo'.

\subsection{Sobre la pérdida de calidad}

Para el cuarto apartado de la encuesta (Causas y razones que van en detrimento de la calidad periodística), además de algunos aspectos aludidos en el anterior epígrafe, relacionados con el contexto socioprofesional, hemos aprovechado los principales argumentos que docena y media de periodistas valencianos le explicaron mediante entrevistas en profundidad a Mónica Parreño en su tesis doctoral. A dichas explicacio- 
nes, que esta investigadora agrupó en ocho campos (Parreño, 2013: 112-113), y que en nuestra encuesta a veces están reformuladas, hemos añadido otros razonamientos para permitir que los 363 periodistas de toda España que han contestado propusieran otras causas de la pérdida de la calidad periodística. Para ello se relacionan la calidad o su ausencia con la precariedad laboral, la crisis económica, la adaptación a las nuevas tecnologías de la información y la comunicación, la brecha generacional o aspectos como la homogeneización de contenidos, la inmediatez o la pérdida de valores.

\section{Análisis de los resultados}

Los resultados de la encuesta constituyen una radiografía de la percepción de los profesionales españoles sobre el trabajo que llevan a cabo, a partir del análisis de las respuestas de 363 periodistas a 45 preguntas que abordan desde aspectos vinculados a la estructura del medio, al tratamiento de la información, el cuidado y esmero en el proceso productivo o el análisis de las razones que influyen en la pérdida de calidad. Los participantes ( $45 \%$ mujeres y $55 \%$ hombres) representan una muestra amplia de la profesión, tanto en lo que respecta a cargos de responsabilidad como a vinculación contractual, medio de comunicación, procedencia geográfica o experiencia en labores de comunicación. En el cuadro que se muestra a continuación se recogen las características de los participantes en la encuesta, tanto en términos porcentuales como en números absolutos:

Tabla 1. Participantes en la encuesta. Fuente: Elaboración propia

\begin{tabular}{|c|c|c|c|c|c|}
\hline \multicolumn{6}{|c|}{$\begin{array}{l}\text { Encuesta online La calidad periodística según la percepción de los periodistas } \\
\text { Fecha de realización: junio y julio } 2013\end{array}$} \\
\hline \multicolumn{6}{|c|}{363 periodistas participantes: $55 \%$ Hombres (198); 45\% Mujeres (165) } \\
\hline \multicolumn{2}{|c|}{$\begin{array}{l}\text { Medios de comunicación en los que } \\
\text { trabajan }\end{array}$} & \multicolumn{2}{|c|}{ Puestos o cargos ocupados } & \multicolumn{2}{|c|}{ Situación contractual } \\
\hline Diario impreso & $31 \%(114)$ & Redactor/a & $58 \%(210)$ & Plantilla & $69 \%(249)$ \\
\hline Televisión & $21 \%(75)$ & $\begin{array}{l}\text { Jefe/a de sección } \\
\text { editor/a }\end{array}$ & $12 \%(45)$ & Colaborador/a & $15 \%(54)$ \\
\hline Diario digital & $18 \%(66)$ & $\begin{array}{lll}\text { Redactor/a } & \text { jefe/a } & \text { o } \\
\text { subdirector/a }\end{array}$ & $7 \%(27)$ & Freelance & $13 \%(48)$ \\
\hline Radio & $14 \%(51)$ & Dirección & $5 \%(18)$ & Becario/a & $3 \%(12)$ \\
\hline Revista impresa & $11 \%(39)$ & Otros & $18 \%(63)$ & & \\
\hline Revista digital & $5 \%(18)$ & & & & \\
\hline
\end{tabular}

\subsection{Relevancia de las informaciones y producción propia}

Los resultados de la encuesta muestran que, entre los propios profesionales, no existe una percepción nítida acerca de la relevancia de los contenidos que publican sus medios. Pese a que este valor constituye uno de los indicadores clave a la hora de evaluar la calidad periodística, solo la mitad de los encuestados $(50 \%)$ considera que el porcentaje de informaciones relevantes es suficiente, mientras que un $28 \%$ las considera escasas y un $23 \%$, elevadas. A la hora de valorar esta opción se observan, sin embargo, algunas diferencias, estrechamente vinculadas a los años de ejercicio profesional. Los recién incorporados (de 3 a 5 años) se muestran menos exigentes que quienes llevan entre 6 y 20 años. Así, mientras solo un 5\% de los primeros considera escaso el número de temas relevantes, la proporción crece hasta un tercio entre los periodistas con una experiencia media (35\%). Para tres de cada cuatro recién incorporados en los últimos 
cinco años (75\%), el nivel de trascendencia de las informaciones es suficiente, una cifra que se reduce de forma importante en opinión de quienes cuentan con 6-12 años (45\%) y 13-20 años (42\%) de experiencia. Sin embargo, entre los profesionales con más de tres décadas en ejercicio, la visión resulta más optimista: un 35\% opina que el interés general es elevado, doce puntos por encima de la media.

Por medios, los de revistas impresas califican como suficiente (70\%) la relevancia de las informaciones, mientras que los periodistas de prensa impresa y digital tienden a polarizarse a la hora de valorarla, con registros similares entre quienes juzgan que es escasa (33\% y $27 \%$, respectivamente) y quienes la considera elevada ( $29 \%$ y $27 \%)$. También resulta interesante destacar que los redactores tienden a ser más críticos que los cargos directivos a la hora de señalar la falta de relevancia, patente para un $34 \%$ de los primeros y apenas evidente para un $23 \%$ del staff. No podemos afirmar, sin embargo, que exista una correlación directa entre el cargo desempeñado y una actitud más o menos crítica hacia las prácticas profesionales, pero sí una cierta tendencia a mirar con severidad aquellas acciones que no dependen directamente de la competencia de una u otra categoría laboral.

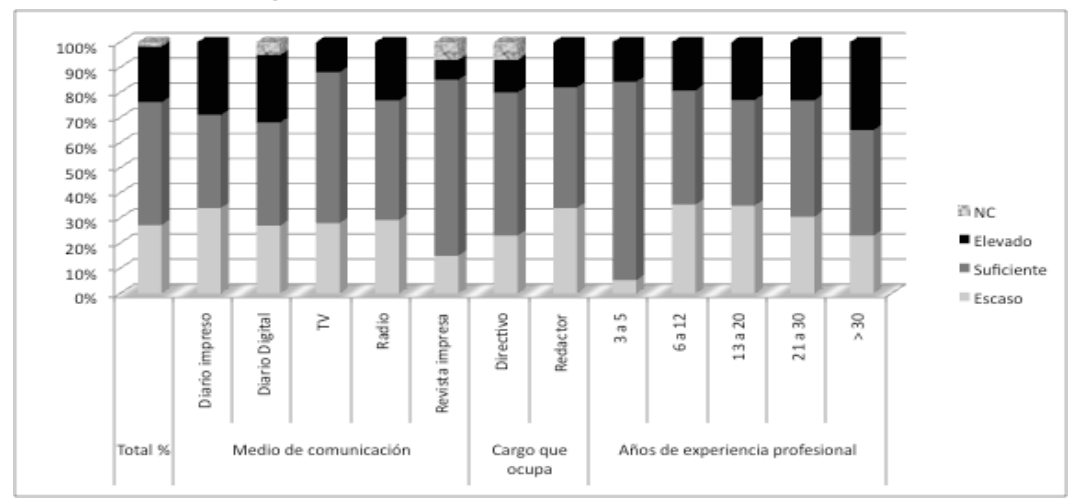

Gráfico 1. Grado de relevancia de las informaciones publicadas en el medio. Fuente: elaboración propia

Casi la mitad de los encuestados coincide a la hora de considerar escaso el porcentaje de temas propios que se publican en sus respectivos medios de comunicación $(48 \%)$, mientras que un $32 \%$ opina que es suficiente y apenas uno de cada cinco (21\%) lo valora como elevado. Las diferencias más destacadas quedan patentes en función de los medios en los que desarrollan su trabajo los entrevistados. De este modo, son los de radio y prensa digital quienes tienen una percepción más negativa sobre la homogeneización de las agendas informativas en sus medios. El 65\% de los primeros considera escaso el porcentaje de temas propios, frente al $11 \%$ que juzga que es elevado. Entre los de diarios digitales, casi seis de cada diez (59\%) creen que la producción propia es limitada, un porcentaje que triplica al de aquellos que sostienen que es elevada (18\%). Si las cifras se comparan con las respuestas de sus homólogos en papel, las diferencias son notables: el 39\% valora como escasos los contenidos propios, mientras que otro $39 \%$ considera que son suficientes, lo que supone 20 y 16 puntos porcentuales respecto a los valores de los periodistas de diarios online. 
Los recién incorporados son, de nuevo, quienes estiman de forma más positiva el porcentaje de producción propia, una opinión que contrasta especialmente con quienes suman más de veinte años en el oficio. Mientras casi un tercio de los profesionales de 3 a 5 años de andadura (30\%) interpreta que los temas propios son elevados, entre los más veteranos esta cifra se reduce casi a la mitad (17\%). Sin embargo, quienes más énfasis ponen en las carencias son los periodistas que cuentan entre 6 y 30 años en el oficio: la mitad de ellos considera que son escasos.

Estas percepciones se repiten con idénticas correlaciones cuando se pregunta ¿cómo podría definir la presencia de reportajes de producción propia en el conjunto de la información que ofrece su medio? Los resultados globales sitúan en un $22 \%$ los profesionales satisfechos con el porcentaje de reportajes propios publicados, mientras que un $45 \%$ lo considera escaso y solo un $33 \%$ lo ve suficiente.

El $63 \%$ de periodistas digitales entiende como limitada la producción de reportajes y un $18 \%$ como suficiente, mientras que para la mitad de quienes desempeñan su actividad en prensa impresa resulta escaso, frente a un 31\% que lo estima suficiente. Junto a los periodistas digitales, son los que trabajan en revistas impresas quienes se muestran más críticos respecto a la escasez de reportajes de producción propia $(61 \%$ de los encuestados), algo que resulta paradójico entre profesionales de un medio de actualidad más relajada. Solo un $8 \%$ responde que es elevado.

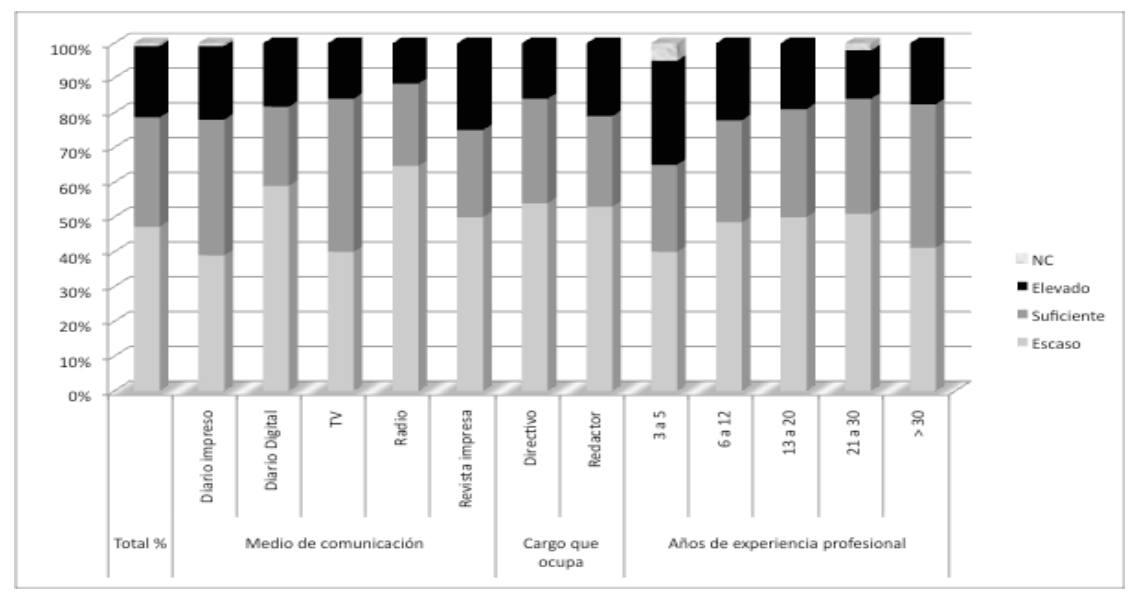

Gráfico 2. Porcentaje de temas propios respecto al total de informaciones Fuente: elaboración propia

La calidad se asocia claramente con la capacidad de ofrecer a los lectores y espectadores contenidos propios, y ello tiene, inevitablemente, unos costes económicos. Estas dos opciones quedan patentes en las respuestas ofrecidas por más de dos tercios de los periodistas y cargos directivos, que consideran que "un medio de calidad debe marcar la diferencia con temas propios" (34\%) y que una buena información necesita de una inversión económica que, en su opinión, "las empresas no están dispuestas a asumir" (34\%). Este dato viene reforzado por la opinión de casi uno de cada cinco encuestados, que subraya que las empresas "no invierten tiempo ni dinero" en buscar nuevas opciones creativas que permitan salir del círculo vicioso de la homo- 
geneización de contenidos, uno de los principales lastres de la calidad. En este sentido, la tendencia a repetir de forma acrítica lo que ha sido útil en otras ocasiones aparece como un factor inversamente proporcional a la posibilidad de alcanzar las cotas profesionales más elevadas en términos de calidad. Sin embargo, el 14\% de las respuestas recabadas destaca que "suelen mimetizarse los temas o coberturas que en otros medios han dado buenos resultados".

\subsection{Investigación, proximidad y fuentes documentales}

Quizás el mejor termómetro para valorar la voluntad de diferenciarse, de ofrecer contenidos que se aparten de la tendencia a la homogeneización denunciada a menudo, sea la presencia de trabajos de investigación en los medios. Sin embargo, los resultados globales no parecen muy alentadores en este sentido, puesto que el $74 \%$ de los encuestados considera que el periodismo de investigación es prácticamente inexistente en sus medios, frente a un $14 \%$ que lo estima suficiente y un $12 \%$ que observa niveles de publicación elevados. Como ocurría en el caso de la producción propia de reportajes, son los periodistas de radio (88\%) y de revistas impresas (84\%) quienes apuntan con mayor intensidad a la práctica inexistencia de trabajos de investigación en sus medios. Los resultados contrastan con la respuesta de los periodistas de diarios digitales, los más críticos a la hora de apuntar a la escasa producción propia: un $27 \%$ considera que es elevada la publicación de estos trabajos, más del doble que la media $(12 \%)$. Aunque el $59 \%$ de profesionales de este medio señala que es casi inexistente, la cifra se sitúa 15 puntos por debajo de la media, lo que muestra una tendencia más positiva que en el resto de medios en la valoración de propuestas que dan a conocer temas de gran interés social que se mantienen ocultos.

Como ocurría en la valoración de la relevancia, la experiencia profesional vuelve a ser un indicador importante en la percepción del periodismo de investigación. Mientras un $29 \%$ de quienes cuentan con más de tres décadas en ejercicio sostiene que la presencia del periodismo de investigación es elevada en sus medios, ninguno de los encuestados que están en el tramo medio ( 6 a 12 años) respalda esta idea. Un 84\% de estos suscribe, además, que es casi inexistente, una tesis que comparten, en porcentajes similares $(81 \%)$, los periodistas que acreditan entre dos y tres décadas de experiencia. Los que se han incorporado de manera reciente, si bien se muestran menos críticos al detectar la escasez -un $60 \%$ cree que es casi inexistente-, tampoco exhiben un gran optimismo, ya que apenas el $5 \%$ de los encuestados de entre 3 y 5 años de experiencia valora como elevada la presencia de periodismo de investigación en el medio en el que trabaja.

Si la presentación de temas propios y la relevancia de estos son factores directamente relacionados con la calidad periodística, no lo es menos el acceso a fuentes primarias para la elaboración de las informaciones. Aunque casi nueve de cada diez encuestados afirma que se recurre a bases documentales primarias ocasionalmente (42\%) o de forma habitual (47\%), existen diferencias interesantes si se analizan los datos en función de los tramos de experiencia, el medio para el que se trabaja o el cargo. Los profesionales con una trayectoria de 6 a 12 años son, de nuevo, quienes se muestran más escépticos a la hora de valorar la frecuencia de estas fuentes en el trabajo periodístico. El 29\% de ellos opina que se emplean a menudo, 18 puntos por debajo de la media. En este caso, la percepción critica es similar a la de los periodistas 
más veteranos y contrasta con el resto de franjas, que consideran -en porcentajes que van del 54 al 59\%- que es frecuente recurrir a las bases documentales primarias. Por medios de comunicación, son los empleados en diarios digitales quienes responden con menos optimismo: solo un tercio opina que es frecuente el uso de estas fuentes -más de 15 puntos por debajo de la media- y un 14\% sostiene que no se usan en su medio. En el extremo contrario, más de la mitad de los periodistas televisivos (52\%) constata que se emplean a menudo y apenas un $4 \%$ niega que se recurra a ellas.

El cargo de responsabilidad ocupado constituye también una variable a tener en cuenta, puesto que frente a casi la mitad de los redactores (48\%) que observa una buena praxis, menos de un tercio de los directores y jefes (30\%) opina que las bases de datos primarias se empleen a menudo. Junto a estos datos, el 10\% de los redactores niega que se recurra a ellas, una cifra que se duplica según la percepción de los cargos de responsabilidad.

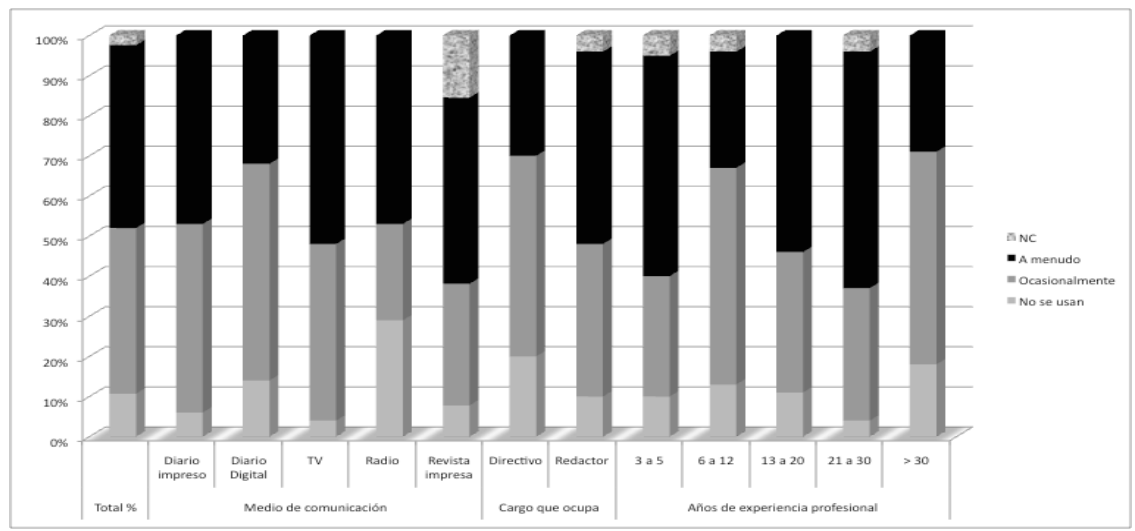

Gráfico 3. Uso de bases documentales primarias en la información del medio. Fuente: Elaboración propia.

Lo que despierta un grado de consenso muy elevado es la tesis de la cercanía que mantienen los medios de comunicación con las realidades más próximas. De este modo, el $68 \%$ cree que casi siempre estas noticias o informaciones próximas predominan sobre las que abordan realidades más lejanas (el $28 \%$ cree que eso sólo se consigue a veces y un $5 \%$, que casi nunca se logra). Este dato, sin embargo, choca con las posibilidades que los periodistas encuentran a la hora de abordar el trabajo informativo, toda vez que no casa con la capacidad de los medios para acceder, de manera directa, a los acontecimientos de los que informa. Así, frente a ese $68 \%$ de quienes sostienen que su medio informativo apuesta por las realidades cercanas en la selección de sus informaciones, solo el $45 \%$ considera que su empresa tiene "mucha" capacidad para cubrirlas de manera directa y el $46 \%$ cree que ello solo se consigue a veces.

Por medios, resulta paradigmático el caso de la radio, ya que si el $94 \%$ de los periodistas asegura que prima la información de proximidad, en cambio, solo un tercio de ellos (35\%) admite tener mucha facilidad para el acceso directo a los acontecimientos de los cuales informa. El mismo desajuste persiste entre la atención de la prensa impresa $(71 \%)$ y la digital $(63 \%)$ a la realidad más cercana y la capacidad de 
conocer de primera mano los hechos. Poco más de una tercera parte de los redactores de diarios digitales (36\%) asegura que tiene muchas posibilidades de acceso a las fuentes primarias, mientras que casi uno de cada cinco asegura que casi nunca tiene medios para llegar a ellas (18\%). Entre los periodistas de medios impresos, solo la mitad afirma tener mucha facilidad, mientras que un $45 \%$ admite que solo a veces. Si se desglosan los datos en función del cargo ocupado, los directores, subdirectores y jefes de sección son menos optimistas: frente al $47 \%$ de periodistas que asegura que su medio tiene mucha capacidad para acceder, solo un $37 \%$ de los cargos de responsabilidad comparte la afirmación.

\subsection{Facticidad y equidad}

Un aspecto clave a la hora de abordar la calidad periodística es conocer el tratamiento de los hechos y las afirmaciones recogidas, así como el esmero para no confundir al lector con meras conjeturas. Por ello resulta sorprendente que la mitad de los encuestados (50\%) afirme que solo "a veces" en las informaciones de su medio los hechos, afirmaciones y conjeturas no aparecen mezclados, mientras que apenas un tercio (32\%) niega con cierta rotundidad esta opción y asegura que no se da "casi nunca". En cambio, casi uno de cada cinco profesionales cree que "casi siempre" aparecen mezcladas. Quienes admiten con más claridad esta mezcla son los periodistas digitales. Un 32\% sostiene que ocurre a menudo en su medio, frente al $6 \%$ de periodistas de radio o el $11 \%$ de prensa impresa. Por años de experiencia profesional, los más sensibles a la hora de ver el problema son los que cuentan con entre una y dos décadas de experiencia. Tres de cada diez (31\%) admite que suele darse "casi siempre" la mezcla de hechos y conjeturas, algo que comparte el $22 \%$ de los colegas con entre 6 y 12 años en ejercicio. Apenas el 11\% de los redactores de 13 a 20 años de experiencia asume que esta práctica no se da "casi nunca". No obstante, son los periodistas más veteranos quienes defienden con mayor vehemencia que esta confusión no se produce "casi nunca", como afirma el 37\% de los de 21-30 años de experiencia y el 53\% de los de más de 30 .

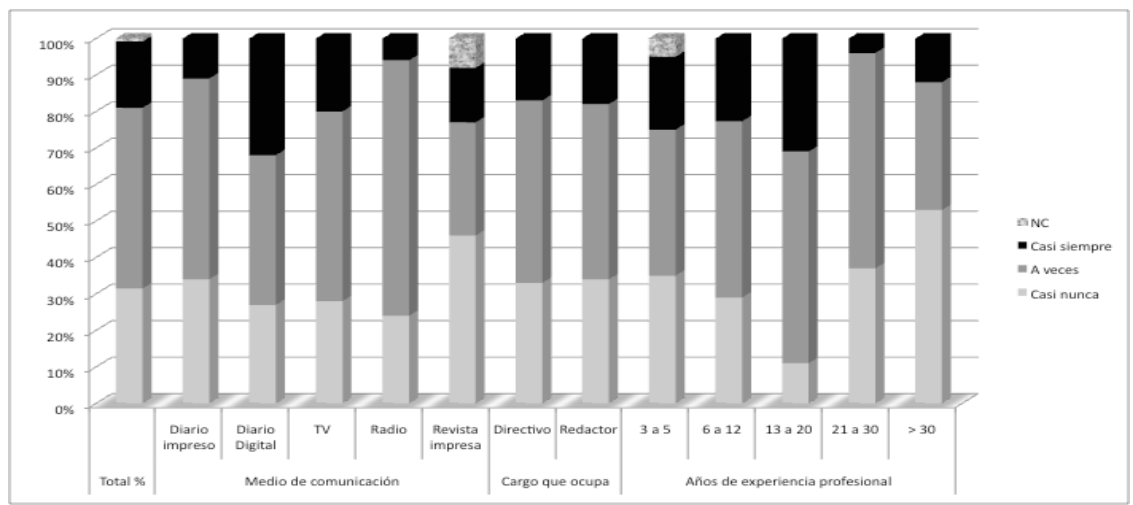

Gráfico 4. Grado de facticidad: hechos y conjeturas no aparecen mezclados. Fuente: elaboración propia 
La mayoría de los encuestados (53\%) considera que en sus respectivos medios existe equidad a la hora de tratar a los protagonistas de las informaciones, si bien un $35 \%$ afirma que esa equidad es "poca" frente al $12 \%$ que la considera "elevada". Una radiografía más detallada de la respuesta permite ver que quienes más acusan este trato poco equilibrado son el $42 \%$ los periodistas de entre 6 y 12 años en ejercicio y el $41 \%$ de los que llevan más de 30 años. Para más de la mitad de profesionales del medio radiofónico (53\%) la equidad es "poca", en la línea de lo que sostiene el $42 \%$ de redactores de medios impresos o el $44 \%$ de televisión, y en claro contraste con el escaso $9 \%$ de periodistas de diarios digitales que detecta estas carencias. Las diferencias en la percepción también son evidentes en función del cargo. Mientras solo un $23 \%$ de los responsables observa la escasa equidad en el tratamiento, un $44 \%$ de redactores la detecta con claridad. Asimismo, frente al $60 \%$ de cargos directivos que la considera suficiente, solo el $47 \%$ de la base redaccional comparte esta visión.

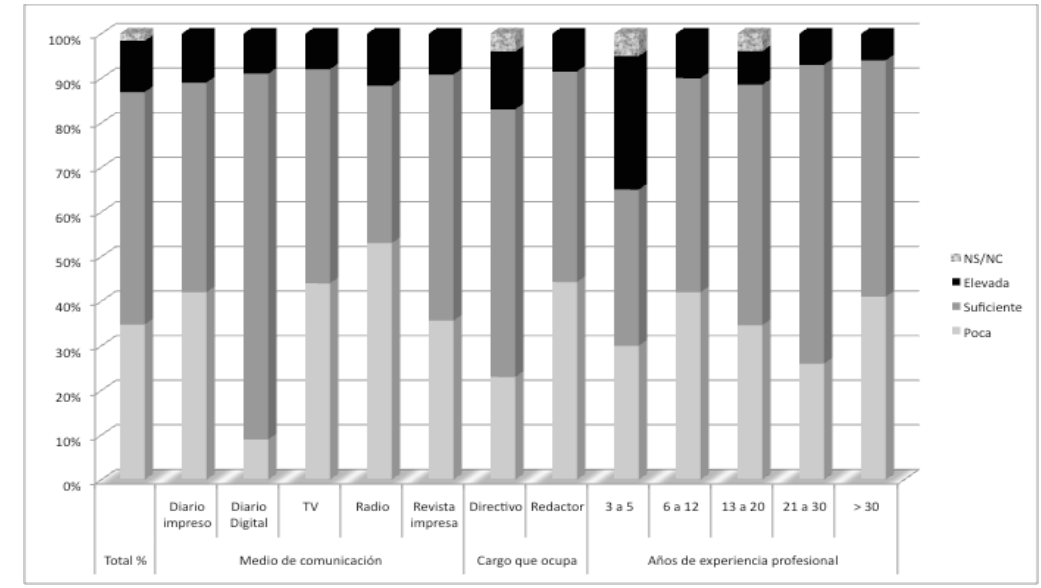

Gráfico 5. Equidad en el tratamiento de los protagonistas de la información. Fuente: elaboración propia.

\subsection{Un contexto laboral poco propicio}

Las circunstancias en las que desarrollan su actividad los periodistas encuestados están lejos de resultar favorables, tanto desde el punto de vista de la jornada laboral como de estabilidad o de salario. Todo ello a pesar del alto grado de profesionalización de las redacciones que constatan los encuestados, ya que el $85 \%$ asegura que el nivel medio de formación de los profesionales de sus medios corresponde al de titulados en Periodismo o en Comunicación Audiovisual. Ese porcentaje llega hasta el 95\% si incluimos al $10 \%$ que sostiene que el perfil medio de su empresa está compuesto por licenciados universitarios en otras titulaciones (un escaso $5 \%$ cree que en su medio imperan los profesionales autodidactas).

Quienes manifiestan que existe en su empresa un alto grado de estabilidad laboral representan el $38 \%$, mientras un $34 \%$ señala que solo la mitad de la plantilla de sus medios goza de dicha estabilidad y casi una tercera parte de los periodistas (28\%) afirma que el nivel de estabilidad es escaso. A estos datos, que reflejan las circuns- 
tancias de crisis por las que pasa el sector, se suman los relativos a las percepciones económicas o las jornadas laborales. El 39\% cree que el nivel salarial es escasamente justo, cifra similar a quienes opinan que el equilibrio trabajo/sueldo se limita a determinados puestos (otro 39\%). Quienes afirman disponer de un salario adecuado a sus funciones suponen un $22 \%$ de los interrogados.

No obstante, como se detalla a continuación, más allá del retrato general de la profesión, pueden observarse algunas realidades particulares. Así, por medios, quienes más acusan la falta de estabilidad laboral son los periodistas de diarios digitales $(50 \%)$, frente a solo un $12 \%$ de los de televisión y el $18 \%$ de los de radio, que aseguran que la estabilidad es escasa. El 46\% de quienes trabajan en revistas impresas y cuatro de cada diez profesionales televisivos sostienen que la estabilidad es alta. La experiencia también es un indicador de la percepción, en especial para los dos extremos temporales: el $40 \%$ de los recién incorporados y el $47 \%$ de los que llevan tres décadas confirman que la estabilidad es escasa, una respuesta en la que pueden leerse los efectos de la dificultad de los recién graduados para encontrar un trabajo no precario y de los más veteranos para no caer en las redes de un ERE. En cambio, quienes parecen más convencidos de la alta seguridad de los puestos de trabajo son los periodistas con 21-30 años (59\%) y 13-20 años en activo (42\%).

Tampoco en lo referido al salario percibido las coincidencias son absolutas cuando se revisan las respuestas en función del medio, el cargo o el número de años en ejercicio. De nuevo son los periodistas digitales quienes inciden en la baja cuantía de los salarios (54\%), mientras que los de televisión son los que perciben un mayor nivel de sueldos adecuados (40\%): en ambos casos, 15 y 18 puntos, respectivamente por encima de la media. Los de prensa impresa, en cambio, ponen el acento en la disparidad y un $50 \%$ de ellos asegura que solo en determinados puestos son justos, mientras que solo para un $11 \%$ resultan adecuados. Los jóvenes periodistas son los más sensibles a la hora de valorar que los sueldos son escasamente justos. El 70\% de los de 3-5 años en ejercicio comulga con esta tesis, que apenas sostiene el $22 \%$ de los periodistas con dos o tres décadas en la profesión. Resulta interesante observar que es el $52 \%$ de estos últimos, junto con el $48 \%$ de los periodistas de 6 a 13 años de trayectoria, quienes perciben con más nitidez que el grado de adecuación del suelto está estrechamente vinculado al puesto de trabajo. Los más satisfechos son, no obstante, los más veteranos: un $35 \%$ de ellos confiesa que los sueldos son adecuados.

El cargo no aparece necesariamente como un indicador de satisfacción con el sueldo o la estabilidad. De hecho, si se comparan las respuestas de los 210 redactores (redactor, redactor gráfico o reportero audiovisual) que han participado con los de los 90 integrantes de equipos de dirección (director/a, subdirector/a y jefe/as de área o jefe/a de sección y editor/a) -el resto de categorías, al que pertenecen desde guionistas a colabores se ha identificado como otros-, son estos últimos los que puntúan de forma menos optimista la situación. El 47\% de los jefes considera que los sueldos del medio en el que trabaja son escasamente justos, mientras que solo un 34\% de los redactores comparte esta misma idea. En la misma línea, solo el 17\% de los cargos de responsabilidad asegura que son adecuados, siete puntos por debajo de la redacción. También a la hora de valorar la estabilidad, el 30\% de los jefes opina que es escasa, una idea que comparte el $24 \%$ de la redacción. 
La categoría laboral pesa poco a la hora de analizar la racionalidad de las jornadas, que un $70 \%$ cree que es poco o relativamente racional en su medio, mientras que solo para uno de cada tres resulta equitativa. Probablemente ello se relacionaría con las diferentes rutinas productivas asociadas a cada tipo de medio. De este modo, el $60 \%$ de quienes dicen tener horarios poco racionales trabajan en diarios impresos, mientras que solo un $12 \%$ de los trabajadores de televisión y un $23 \%$ de los radiofónicos perciben este mismo problema. Por franjas de experiencia laboral, destaca la crítica del $45 \%$ de los periodistas con entre 13 y 20 años de carrera, que valoran como poco racionales los horarios de trabajo, lo que implica 27 puntos de diferencia sobre la percepción de los más veteranos.

Tanto las respuestas a la pregunta anterior como la constatación de escasez de temas propios se relacionan con unas plantillas que para casi siete de cada diez encuestados $(69 \%)$ son insuficientes, para el $26 \%$ resultan suficientes y para un escaso $5 \%$, elevadas. En este sentido, son los redactores de medios digitales (82\%) e impresos $(74 \%)$ quienes observan nítidamente que el número de redactores de los que dispone su medio en relación con el volumen de trabajo total es escaso. Por franjas de experiencia, los más críticos son los profesionales de entre 6 y 12 años. Ocho de cada diez encuestados asegura que la proporción resulta escasa, mientras que solo el 52\% de los que tienen de dos a tres décadas de experiencia comparte esta percepción.

Cuando surgen temas de relevancia que exigen un tratamiento intensivo, los medios suelen destinar, según la mitad de los encuestados, de dos a tres redactores a cubrirlos. Sin embargo, más de un tercio de los periodistas (36\%) asegura que apenas se hace cargo de ellos una persona, mientras que el 13\% afirma que en estas situaciones pueden realizar el seguimiento de un tema equipos de cuatro o cinco personas.

\subsection{Adaptación a las nuevas tecnologías e inmediatez}

La adaptación a las nuevas tecnologías plantea retos importantes a los periodistas, en particular, como reseña casi uno de cada cuatro entrevistados (23\%), la falta de tiempo para reflexionar sobre la información, el factor que más pesa en la rebaja de la calidad. El 15\% de los periodistas achaca la merma a los actuales modos de producción, algo que, en su opinión, aumenta los errores, un porcentaje similar (14\%) al que suscribe la idea de que las nuevas tecnologías ofrecen más medios, pero ello contrasta con una "menor capacidad para cubrir los contenidos", lo que perjudica la calidad.

Algunas de las causas de la relajación de los estándares profesionales asociadas a las nuevas tecnologías se relacionan, precisamente, con la transformación de los modos de trabajo. Así, un $24 \%$ considera que persiste una brecha importante entre la redacción tradicional y la digital, o echa en falta una mejor adaptación y aprendizaje sobre cómo hacer periodismo con los nuevos dispositivos. Las redes sociales constituyen, en cierta medida, un motivo de alerta, puesto que un $12 \%$ de los encuestados valora que, en la merma de calidad, influye el hecho de que "el periodismo está compitiendo" con ellas, al que se suma un 5\% que opina que el tiempo destinado a contenidos para ellas "repercute negativamente en la calidad". Junto a estas apreciaciones, un $6 \%$ de los encuestados afirma que la coexistencia de diversos modos de publicación o difusión del medio lastra el trabajo informativo. De las ocho respuestas libres 
que ha generado la pregunta sobre la adaptación a las nuevas tecnologías, cinco han sido para negar que estas tecnologías tengan efectos negativos, mientras que las tres restantes se sustentan en los siguientes argumentos: "Los responsables de contenidos digitales tiene[n] muy poco nivel periodístico", "Se pierde el contacto periodista - informador" o "Sólo afecta a los medios tradicionales, que deberían reforzar sus plantillas".

Cuando se pregunta por las repercusiones de la exigencia de inmediatez sobre la calidad, destacan dos argumentos que suscriben cuatro de cada diez periodistas: la percepción de que la falta de tiempo impide la profundización en los contenidos (22\%) y la idea de que el afán de instantaneidad es la causa del aumento de errores graves en las informaciones (18\%). Junto a ellos destaca también la inquietud sobre el tipo de contenidos que se ofrecen. Si bien prima la responsabilidad atribuida a la empresa -como segundo responsable de la merma de calidad ligada a la inmediatez-, en algunos casos también se aprecia cierta autocrítica. Así, el 18\% de las respuestas coincide en señalar que "la empresa periodística ni se preocupa mucho ni insiste suficientemente en cuidar el contenido", al que se suma otro 9\% que critica la escasa selectividad de lo que se presenta como noticia. No obstante, algunas de las respuestas subrayan que los fallos de precisión que impiden ofrecer un producto de calidad son, en sentido amplio, "el resultado de malos hábitos diversos" (11\%), mientras que casi uno de cada diez encuestados asegura que "los periodistas descuidan a menudo el contenido y la forma de presentar la información".

\section{Conclusiones}

La fotografía que se desprende de la percepción de los profesionales sobre la calidad periodística de los medios muestra algunas carencias importantes, relativas tanto a la relevancia y diversidad de los temas abordados como al acceso a las fuentes o el tratamiento y verificación de los datos, así como a las condiciones laborales o a los retos derivados de la adaptación a las nuevas tecnologías y a las imposiciones de la acuciante inmediatez. Sin embargo, más allá de la imagen de conjunto, persisten algunas circunstancias que se manifiestan de manera más intensa en función del medio en el que se trabaja, de los años de experiencia o del cargo que se ocupa.

Pese a que la mitad de los encuestados no da más que un "suficiente" a la relevancia de los temas que publica su medio y un $48 \%$ opina que el porcentaje de información propia es escaso, las respuestas muestran puntos de divergencia en función de factores como la experiencia. Son los nuevos periodistas, los que cuentan entre 3 y 5 años de trayectoria, quienes se muestran más complacientes con ambos aspectos (solo un 5\% ve escasa la presencia de temas importantes y el 30\% considera elevada la de informaciones propias). Quizás la escasa perspectiva pesa en una valoración que se vuelve mucho más autocrítica entre los profesionales de 6 a 12 años, en la mayoría de casos en clara coincidencia con los que conformarían el grupo de 13 a 20 años, es decir, los de trayectoria media. Esta franja de veteranía es, sin duda, la que muestra una visión menos optimista de buena parte de los criterios sobre los que se asienta una práctica periodística de calidad (acceso a las fuentes primarias, apuesta por el periodismo de investigación, mezcla de hechos y conjeturas o equidad en el tratamiento de 
los protagonistas de las informaciones). En cambio, entre los decanos con más de 30 años de trayectoria suele pesar cierta displicencia a la hora de ver el vaso medio lleno en asuntos como el elevado porcentaje de temas relevantes o propios, aunque, en cambio, muestran un mayor convencimiento acerca de la apuesta actual por el periodismo de investigación, que duplica con creces la percepción de la media de participantes en la encuesta.

El medio en que se desarrolla la labor profesional constituye un filtro importante a tener en cuenta. Los más críticos en cuanto a presencia de temas propios, reportajes e investigación son los periodistas de radio y los de revistas impresas, algo que choca con el modelo de estos últimos. Junto a ellos son los periodistas de diarios digitales quienes muestran mayor descontento con los temas propios y la escasez de reportajes, pero, paradójicamente, los profesionales de medios online son quienes defienden con más optimismo la elevada presencia de investigación (un 27\%, frente al 12\% de media).

A pesar de que los encuestados aseguran que en sus medios la proximidad prima sobre la información de realidades lejanas (68\%), en el caso de la radio y la prensa impresa se produce un fenómeno contradictorio, ya que, si bien superan el índice medio de cobertura de temas locales, muestran poca capacidad para acceder de manera directa a los acontecimientos, algo que, en menor medida, afecta también a los periodistas digitales, los que más dificultad tienen, junto con los de revistas impresas, para superar este escollo. En lo que respecta al uso de fuentes documentales primarias, son de nuevo los profesionales del medio radiofónico quienes aseguran con más contundencia que no se usan (en un 29\%, frente a una media del 11\%). Cuando se plantean cuestiones como la facticidad y la equidad, los periodistas de radio y diarios digitales se convierten en protagonistas de tendencias desiguales. Si en el primer caso son los periodistas online quienes admiten más dificultades (un 32\% asegura que hechos y conjeturas se mezclan casi siempre, casi el doble de la media), en el segundo son los de radio quienes aparecen como los menos equilibrados en el tratamiento de los protagonistas (un 53\% afirma que es poca la equidad).

La responsabilidad ejercida en el medio influye de manera desigual en la percepción de los encuestados, ya que, más que marcar una predisposición a la crítica, pondera la visión sobre unos u otros comportamientos, en ocasiones con diferencias importantes. En general se observa la tendencia a una mayor o menor severidad en el juicio en función de la competencia laboral directa sobre el aspecto en cuestión. Así, los jefes tienden a ser menos estrictos que sus subordinados a la hora de valorar la falta de relevancia de los temas publicados (un 23\%, frente al $34 \%$ de los redactores), la escasa equidad en el tratamiento de los protagonistas (un 23\%, frente al $42 \%$ de la redacción) o la dificultad para acceder de manera directa a los acontecimientos (un $37 \%$, frente a un $47 \%$ de los redactores), mientras que los directores, jefes de sección o editores admiten de modo más abierto que el uso de fuentes primarias es bastante inferior a lo reconocido por los periodistas (un 30\%, frente al $48 \%$ que aseguran estos últimos). Cuando se trata de valorar las condiciones de trabajo, es el staff directivo quien alza la voz reivindicativa: el $47 \%$ de sus integrantes asegura que el sueldo es escasamente justo (ocho puntos por encima de la media y 13 más sobre los redactores) y el $30 \%$ considera la estabilidad escasa (seis puntos más que los periodistas). 
La precariedad laboral se expresa de manera clara en función de la edad y del medio en el que desarrollan su actividad los periodistas. El perfil de los más afectados, tanto en materia de estabilidad como de salario, es el de los empleados en medios digitales con menos de cinco años de experiencia, aunque también los de más de tres décadas en ejercicio apuntan a una escasa estabilidad (un 47\%, veinte puntos por encima de la media). No obstante, estos últimos son los que mejor puntúan la opción de sueldos adecuados. La televisión, por su parte, parece el medio que ofrece una mayor seguridad y salarios más adecuados (un $40 \%$, casi el doble de la media), mientras que la prensa impresa - con niveles de estabilidad medios- se muestra como la que más tiende a condicionar la equidad o no de los sueltos al puesto ocupado; también esta última acoge a quienes más acusan jornadas poco racionales.

Los participantes en la encuesta tienden a dejar caer sobre el tejado de las empresas buena parte de la iniciativa para resolver los problemas de calidad, en especial a aludir a la falta de inversión para mejorar las condiciones de producción y poder optar por temas propios y tratamientos originales frente a la homogeneización de contenidos y el mimetismo. Buena parte de los encuestados considera que la adaptación a las nuevas tecnologías pesa sobre los tiempos y modos de producción, como la exigencia de inmediatez, que impide profundizar en los temas y genera numerosos errores, circunstancias que influyen directamente en la merma de calidad.

\section{Referencias bibliográficas}

ALSIUS, Salvador y SALGADO, Francesc (eds.) (2010): La ética informativa vista por los ciudadanos. Contraste de opiniones entre los periodistas y el público. Barcelona, UOC.

BOGART, Leo (1989): Press and Public. Who reads what, when, where, and why in American Newspapers. Hillsdale, L. Erlbaum Ass.

CIS (2013): Barómetro de marzo. Estudio $\mathrm{n}^{\circ}$ 2.981, en: http://datos.cis.es/pdf /Es2981mar_A.pdf [fecha de consulta: 15 de abril de 2014].

De PABLOS COELLO, José Manuel y MATEOS MARTÍN, Concha (2004): "Estrategias informativas para acceder a un periodismo de calidad en prensa y TV". Ámbitos, $\mathrm{n}^{\mathrm{o}}$ 11-12, pp. 341-365.

GÓMEZ MOMPART, Josep Lluís y PALAU SAMPIO, Dolors (2013): "Métodos y técnicas de análisis y registro para investigar la calidad periodística". Comunicación presentada en el II Congreso Nacional de Metodología de la investigación en comunicación de la AEIC, Segovia.

GÓMEZ MOMPART, Josep Lluís; GUTIÉRREZ LOZANO, Juan Francisco y PALAU SAMPIO, Dolors (eds.) (2013): La calidad periodística. Teorías, investigaciones y sugerencias profesionales. Barcelona, Castellón y Valencia, UAB, UPF, UJI y UV.

GONZÁLEZ, María (2011): Albisteen kalitatea. Euskadi Irratia, Etb1 eta Euskaldunon Egunkaria/Berria (Research on Basque Media's News Quality). Tesis doctoral. Bilbao, Universidad del País Vasco. 
ISRAEL GARZÓN, Estrella y POMARES PASTOR, Ricardo (2013): “Indicadores de calidad en los informativos de televisión". En GÓMEZ MOMPART, Josep Lluís; GUTIÉRREZ LOZANO, Juan Francisco y PALAU SAMPIO, Dolors (eds.): $L a$ calidad periodística. Teorias, investigaciones y sugerencias profesionales. Barcelona, Castellón y Valencia, UAB, UPF, UJI y UV, pp. 147-161.

MERRILL, John y LOWENSTEIN, Ralph (1971): Media, messages, and men: New perspectives in communication. Nueva York, D. McKay Co.

PARREÑO RABADÁN, Mònica (2013): "La argumentación de los periodistas sobre la calidad del periodismo". En GÓMEZ MOMPART, Josep Lluís; GUTIÉRREZ LOZANO, Juan Francisco y PALAU SAMPIO, Dolors (eds.): La calidad periodística. Teorías, investigaciones y sugerencias profesionales. Barcelona, Castellón y Valencia, UAB, UPF, UJI y UV, pp. 105-118.

TÉRAMO, María Teresa (2006): “Calidad de la información periodística en Argentina. Estudio de diarios y noticieros". Palabra Clave, vol. 9 (1), pp. 57-84.

VVAA (2001): "VAP: Un sistema métrico de la calidad periodística". Cuadernos de Información, $\mathrm{n}^{\mathrm{o}}$ 14, pp. 112-120.

VVAA (2006): Propuesta de indicadores para un periodismo de calidad en México, en: $\mathrm{http} / /$ prende.masimagen.com.mx/images/stories/file/librodigitalpropuestaindicadoresperiodismocalidadmexico.pdf [fecha de consulta: 15 de abril de 2014]. 